\title{
Sensitivity of the NEXT experiment to Xe-124 double electron capture
}

\section{Onext}

\section{The NEXT collaboration}

G. Martínez-Lema, ${ }^{s, u, 1}$ M. Martínez-Vara, ${ }^{s, p}$ M. Sorel, ${ }^{s}$ C. Adams, ${ }^{b}$ V. Álvarez, ${ }^{v}$ L. Arazi, ${ }^{f}$ I.J. Arnquist, ${ }^{t}$ C.D.R Azevedo, ${ }^{d}$ K. Bailey, ${ }^{b}$ F. Ballester, ${ }^{v}$ J.M. Benlloch-Rodríguez, ${ }^{p, s}$ F.I.G.M. Borges, ${ }^{n}$ N. Byrnes, ${ }^{c}$ S. Cárcel, ${ }^{s}$ J.V. Carrión, ${ }^{s}$ S. Cebrián, ${ }^{w}$ E. Church, ${ }^{t}$ C.A.N. Conde, ${ }^{n}$ T. Contreras, ${ }^{k}$ G. Díaz, ${ }^{u}$ J. Díaz, ${ }^{s}$ M. Diesburg, ${ }^{e}$ J. Escada, ${ }^{n}$ R. Esteve, ${ }^{v}$ R. Felkai, ${ }^{f, g, s}$ A.F.M. Fernandes, ${ }^{m}$ L.M.P. Fernandes, ${ }^{m}$ P. Ferrario, ${ }^{p, i}$ A.L. Ferreira, ${ }^{d}$ E.D.C. Freitas, ${ }^{m}$ J. Generowicz, ${ }^{p}$ S. Ghosh, ${ }^{k}$ A. Goldschmidt, ${ }^{h}$ J.J. Gómez-Cadenas, ${ }^{p, i, 2}$ D. González-Díaz, ${ }^{u}$ R. Guenette, ${ }^{k}$ R.M. Gutiérrez, ${ }^{j}$ J. Haefner, ${ }^{k}$ K. Hafidi, ${ }^{b}$ J. Hauptman, ${ }^{a}$ C.A.O. Henriques, ${ }^{m}$ J.A. Hernando Morata, ${ }^{u}$ P. Herrero, ${ }^{p}$ V. Herrero, ${ }^{v}$ Y. Ifergan, ${ }^{f, g}$ S. Johnston, ${ }^{b}$ B.J.P. Jones, ${ }^{c}$ M. Kekic, ${ }^{u, s}$ L. Labarga, ${ }^{r}$ A. Laing, ${ }^{c}$ P. Lebrun, ${ }^{e}$ N. López-March, ${ }^{v, s}$ M. Losada, ${ }^{j}$ R.D.P. Mano, ${ }^{m}$ J. Martín-Albo, ${ }^{s}$ A. Martínez, ${ }^{s, p}$ A.D. McDonald, ${ }^{c}$ F. Monrabal, ${ }^{p, i}$ C.M.B. Monteiro, ${ }^{m}$ F.J. Mora, ${ }^{v}$ J. Muñoz Vidal, ${ }^{s, p}$ P. Novella, ${ }^{s}$ D.R. Nygren, ${ }^{c, 2}$ B. Palmeiro, ${ }^{u, s}$ A. Para, ${ }^{e}$ J. Pérez, ${ }^{l}$ M. Querol, ${ }^{s}$ A.B. Redwine, ${ }^{f}$ J. Renner, ${ }^{u, s}$ J. Repond, ${ }^{b}$ S. Riordan, ${ }^{b}$ L. Ripoll, ${ }^{q}$ Y. Rodríguez García, ${ }^{j}$ J. Rodríguez, ${ }^{v}$ L. Rogers, ${ }^{c}$ B. Romeo, ${ }^{p, l}$ C. Romo-Luque, ${ }^{s}$ F.P. Santos, ${ }^{n}$ J.M.F. dos Santos, ${ }^{m}$ A. Simón, ${ }^{f}$ C. Sofka, ${ }^{o, 3}$ T. Stiegler, ${ }^{o}$ J.F. Toledo, ${ }^{v}$ J. Torrent, ${ }^{p}$ A. Usón, ${ }^{s}$ J.F.C.A. Veloso, ${ }^{d}$ R. Webb, ${ }^{o}$ R. Weiss-Babai, ${ }^{f, 4}$ J.T. White, ${ }^{o, 5}$ K. Woodruff ${ }^{c}$ and N. Yahlali ${ }^{s}$

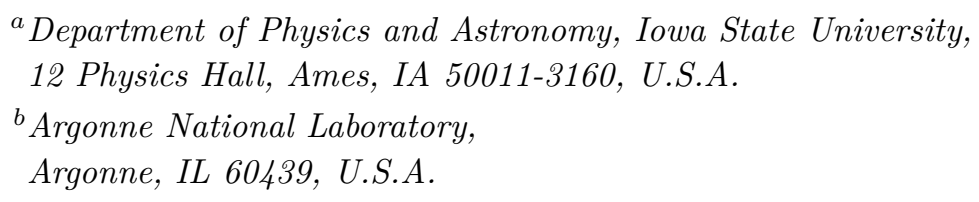

\footnotetext{
${ }^{1}$ Corresponding author. Now at Weizmann Institute of Science, Israel.

${ }^{2}$ NEXT Co-spokesperson.

${ }^{3}$ Now at University of Texas at Austin, U.S.A.

${ }^{4}$ On leave from Soreq Nuclear Research Center, Yavneh, Israel.

${ }^{5}$ Deceased.
} 
${ }^{c}$ Department of Physics, University of Texas at Arlington, Arlington, TX 76019, U.S.A.

${ }^{d}$ Institute of Nanostructures, Nanomodelling and Nanofabrication (i3N), Universidade de Aveiro, Campus de Santiago, Aveiro 3810-193, Portugal

${ }^{e}$ Fermi National Accelerator Laboratory,

Batavia, IL 60510, U.S.A.

${ }^{f}$ Nuclear Engineering Unit, Faculty of Engineering Sciences, Ben-Gurion University of the Negev, P.O.B. 653, Beer-Sheva 8410501, Israel

${ }^{g}$ Nuclear Research Center Negev, Beer-Sheva 84190, Israel

${ }^{h}$ Lawrence Berkeley National Laboratory (LBNL),

1 Cyclotron Road, Berkeley, CA 94720, U.S.A.

${ }^{i}$ Ikerbasque, Basque Foundation for Science,

Bilbao E-48013, Spain

${ }^{j}$ Centro de Investigación en Ciencias Básicas y Aplicadas, Universidad Antonio Nariño, Sede Circunvalar, Carretera 3 Este No. 47 A-15, Bogotá, Colombia

${ }^{k}$ Department of Physics, Harvard University, Cambridge, MA 02138, U.S.A.

${ }^{l}$ Laboratorio Subterráneo de Canfranc,

Paseo de los Ayerbe s/n, Canfranc Estación E-22880, Spain

${ }^{m}$ LIBPhys, Physics Department, University of Coimbra,

Rua Larga, Coimbra 3004-516, Portugal

${ }^{n}$ LIP, Department of Physics, University of Coimbra,

Coimbra 3004-516, Portugal

${ }^{\circ}$ Department of Physics and Astronomy, Texas A\&M University, College Station, TX 7r843-4242, U.S.A.

${ }^{p}$ Donostia International Physics Center (DIPC),

Paseo Manuel Lardizabal, 4, Donostia-San Sebastian E-20018, Spain

${ }^{q}$ Escola Politècnica Superior, Universitat de Girona,

Av. Montilivi, s/n, Girona E-17071, Spain

${ }^{r}$ Departamento de Fúsica Teórica, Universidad Autónoma de Madrid, Campus de Cantoblanco, Madrid E-28049, Spain

sInstituto de Física Corpuscular (IFIC), CSIC \& Universitat de València, Calle Catedrático José Beltrán, 2, Paterna E-46980, Spain

${ }^{t}$ Pacific Northwest National Laboratory (PNNL),

Richland, WA 99352, U.S.A.

${ }^{u}$ Instituto Gallego de Física de Altas Energías, Universidad de Santiago de Compostela, Campus Sur, Rúa Xosé María Suárez Núñez, s/n, Santiago de Compostela E-15782, Spain

${ }^{v}$ Instituto de Instrumentación para Imagen Molecular (I3M), Centro Mixto CSIC, Universitat Politècnica de València, Camino de Vera s/n, Valencia E-46022, Spain

${ }^{w}$ Centro de Astropartículas y Física de Altas Energías (CAPA), Universidad de Zaragoza, Calle Pedro Cerbuna, 12, Zaragoza E-50009, Spain

E-mail: gonzalo.martinez.lema@weizmann.ac.il 
ABSTRACT: Double electron capture by proton-rich nuclei is a second-order nuclear process analogous to double beta decay. Despite their similarities, the decay signature is quite different, potentially providing a new channel to measure the hypothesized neutrinoless mode of these decays. The Standard-Model-allowed two-neutrino double electron capture $(2 \nu E C E C)$ has been predicted for a number of isotopes, but only observed in ${ }^{78} \mathrm{Kr},{ }^{130} \mathrm{Ba}$ and, recently, ${ }^{124} \mathrm{Xe}$. The sensitivity to this decay establishes a benchmark for the ultimate experimental goal, namely the potential to discover also the lepton-number-violating neutrinoless version of this process, $0 \nu E C E C$. Here we report on the current sensitivity of the NEXT-White detector to ${ }^{124} \mathrm{Xe} 2 \nu E C E C$ and on the extrapolation to NEXT-100. Using simulated data for the $2 \nu E C E C$ signal and real data from NEXT-White operated with ${ }^{124} \mathrm{Xe}$-depleted gas as background, we define an optimal event selection that maximizes the NEXT-White sensitivity. We estimate that, for NEXT-100 operated with xenon gas isotopically enriched with $1 \mathrm{~kg}$ of ${ }^{124} \mathrm{Xe}$ and for a 5 -year run, a sensitivity to the $2 \nu E C E C$ half-life of $6 \times 10^{22}$ y (at $90 \%$ confidence level) or better can be reached.

Keywords: Dark Matter and Double Beta Decay (experiments)

ARXIV EPRINT: 2006.07320 


\section{Contents}

1 Introduction 1

2 The NEXT experimental program 3

3 Data samples $\quad 5$

3.1 Background data sample 5

$\begin{array}{lc}3.2 & \text { Signal simulation sample }\end{array}$

4 Event selection $\quad 7$

$4.1{ }^{83 \mathrm{~m}} \mathrm{Kr}$ veto requirement 8

4.2 Data quality selections 9

$\begin{array}{lll}4.3 & \text { Fiducial selection } & 10\end{array}$

$\begin{array}{lll}4.4 & \text { Event energy selection } & 11\end{array}$

4.5 Multi-site selection 11

$\begin{array}{lll}4.6 & \text { Event selection summary } & 12\end{array}$

5 Sensitivity and precision projections $\quad 13$

$\begin{array}{lll}5.1 & \text { Nominal sensitivity and precision } & 15\end{array}$

$\begin{array}{ll}5.2 \text { Potential improvements } & 15\end{array}$

6 Conclusions 18

\section{Introduction}

Ever since the discovery of neutrino oscillations [1-3], and hence neutrino mass, there has been a sustained interest in searches for neutrinoless double beta decay processes. Such second-order weak interactions are generally considered to be the most promising way to test whether neutrinos are massive Majorana particles, identical to their anti-particles. Four related double beta processes have been proposed [4]. The neutrinoless double $\beta^{-}$ emission process $\left(0 \nu \beta \beta,(A, Z) \rightarrow(A, Z+2)+2 e^{-}\right)$is possible in neutron-rich nuclei. This is, by far, the double beta process that has been explored the most both from an experimental and from a theoretical point of view. In proton-rich nuclei, up to three competing processes may be kinematically available: the neutrinoless modes of double $\beta^{+}$emission, single $\beta^{+}$emission plus single electron capture, and double electron capture $(0 \nu E C E C$, $\left.(A, Z)+2 e^{-} \rightarrow(A, Z-2)\right)$. Despite the much lower isotopic abundances of proton-rich nuclei undergoing double beta processes, $0 \nu E C E C$ may provide an interesting alternative to $0 \nu \beta \beta$ searches. Particular interest in $0 \nu E C E C$ has been triggered by the special case 

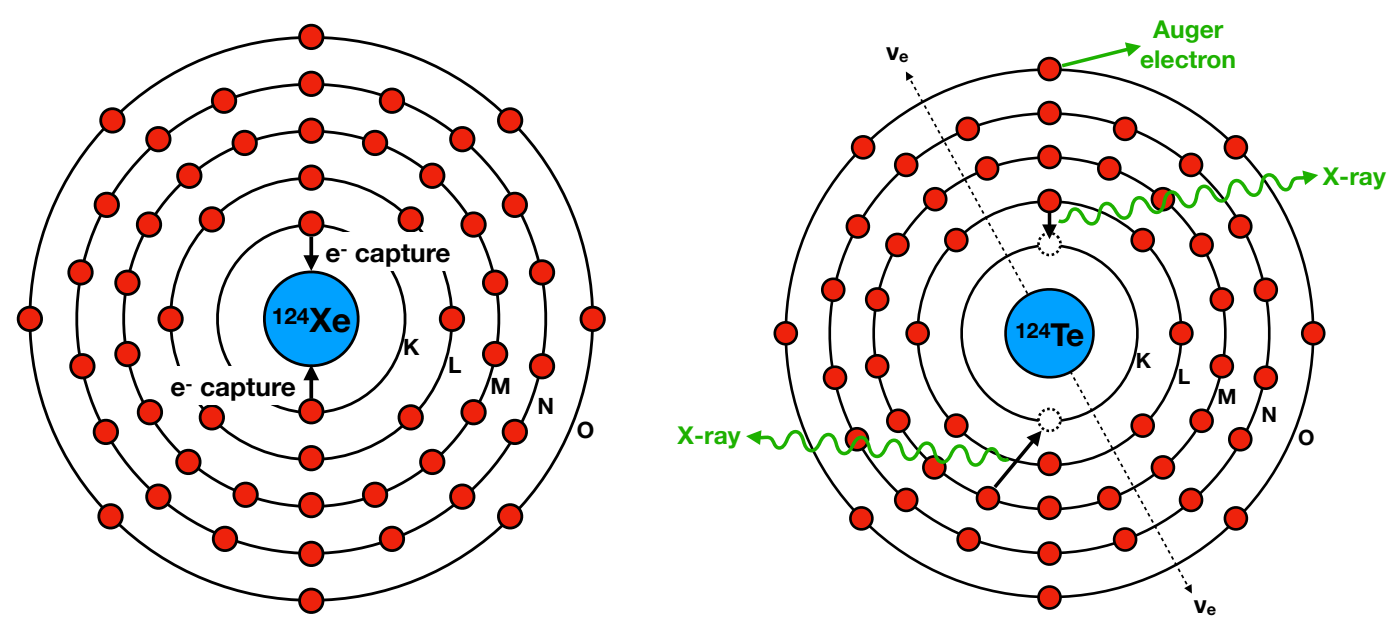

Figure 1. Schematic of the $2 \nu E C E C$ process in ${ }^{124} \mathrm{Xe}$. Left: two orbital electrons from the $\mathrm{K}$ shell are captured by the ${ }^{124} \mathrm{Xe}$ nucleus. Right: the resulting ${ }^{124} \mathrm{Te}$ atom de-excites via X-ray emission (two quanta in this example, from $\mathrm{L} \rightarrow \mathrm{K}$ and $\mathrm{M} \rightarrow \mathrm{K}$ transitions, respectively) or Auger electrons from the outer shells. The two electron neutrinos produced in the nuclear capture escape undetected.

in which the energy of the initial state matches precisely the energy of the (excited) final state $[5,6]$. If this resonance condition is met, the $0 \nu E C E C$ rate is expected to be increased by several orders of magnitude compared to the non-resonant (radiative) case.

A fundamental step toward sensitive $0 \nu E C E C$ searches is the unambiguous measurement of the two-neutrino double electron capture $\left(2 \nu E C E C,(A, Z)+2 e^{-} \rightarrow(A, Z-2)+\right.$ $\left.2 \nu_{e}\right)$. This process is allowed in the Standard Model of particle physics, yet it is predicted to be extremely rare. The $2 \nu E C E C$ predictions use similar many-body techniques and nuclear models as the ones employed for $0 \nu E C E C$. Hence, a measurement of $2 \nu E C E C$ rates is also relevant for interpreting $0 \nu E C E C$ results. In $2 \nu E C E C$, the excess energy is largely carried away by the two undetected neutrinos, with the recoil nucleus kinetic energy being too low $(\sim 10 \mathrm{eV})$ to be detected either. The experimental signature is hence solely given by a cascade of X-rays and Auger electrons. This cascade is a consequence of the readjustment of the electron configuration of the atom that follows the capture by the nucleus of the two orbital electrons, typically from the $\mathrm{K}$ shell. The signature therefore lies in the tens of keV energy range, with all X-rays and Auger electrons produced at the same spatial location. A schematic representation of this process is shown in figure 1, taking capture in ${ }^{124} \mathrm{Xe}$ as an example.

Three experimental indications of $2 \nu E C E C$ exist to date. A possible evidence for $E C E C$ has been reported in ${ }^{130} \mathrm{Ba}$ from geochemical measurements of the ${ }^{130} \mathrm{Xe}$ daughter, with half-life values in the $(5-30) \times 10^{20} \mathrm{yr}$ range $[11,12]$. However, the half-lives of the two quoted ${ }^{130} \mathrm{Ba}$ measurements are incompatible with each other. In addition, these measurements cannot separate possible contributions from the three competing double beta decay processes mentioned above, nor contributions from two-neutrino and neutrinoless modes. An indication with $4 \sigma$ significance has been reported for direct evidence of $2 \nu E C E C$ in ${ }^{78} \mathrm{Kr}$ with a gas proportional chamber, with a half-life of 


\begin{tabular}{|lcccc|}
\hline Experiment & $\begin{array}{c}124 \\
\text { Xe mass } \\
(\mathrm{kg})\end{array}$ & $\begin{array}{c}\text { Live time } \\
(\mathrm{yr})\end{array}$ & $\begin{array}{c}\text { Exposure } \\
(\mathrm{kg} \cdot \mathrm{yr})\end{array}$ & $\begin{array}{c}T_{1 / 2} \\
\left(10^{22} \mathrm{yr}\right)\end{array}$ \\
\hline XENON100 [7] & 0.029 & 0.615 & 0.018 & $>0.07$ \\
XMASS-I [8] & 0.311 & 2.190 & 0.681 & $>2.1$ \\
Gavriljuk et al. [9] & 0.059 & 1.760 & 0.103 & $>0.77$ \\
XENON1T [10] & 1.493 & 0.487 & 0.726 & $1.8 \pm 0.5$ \\
\hline
\end{tabular}

Table 1. Summary of ${ }^{124} \mathrm{Xe} 2 \nu E C E C$ results, for electrons captured from the K shell. For each experiment, the mass of ${ }^{124} \mathrm{Xe}$ in the fiducial volume, the live time, the exposure and the half-life result are given. Lower limits are given at $90 \%$ confidence level.

$T_{1 / 2}\left({ }^{78} \mathrm{Kr}\right)=\left(1.9_{-0.7}^{+1.3} \pm 0.3\right) \times 10^{22} \mathrm{yr}[13]$. Recently, an observation with $4.4 \sigma$ significance has also been reported in ${ }^{124} \mathrm{Xe}$ by the XENON1T collaboration using a detector originally designed for direct dark matter searches, with $T_{1 / 2}\left({ }^{124} \mathrm{Xe}\right)=(1.8 \pm 0.5 \pm 0.1) \times 10^{22} \mathrm{yr}[10]$. Details of recent $2 \nu E C E C$ searches in ${ }^{124} \mathrm{Xe}$ are given in table 1 . Such searches have been performed either using large liquid xenon detectors $[7,8,10]$ with a ${ }^{124}$ Xe isotopic abundance close to the one of natural xenon $\left(9.52 \times 10^{-4}[14]\right)$, or using gas proportional counters using ${ }^{124}$ Xe-enriched xenon [9]. Recent predictions from nuclear structure calculations [15-17] show good agreement with the ${ }^{124}$ Xe half-life measurement by XENON1T. In ${ }^{124} \mathrm{Xe}$, a $76.7 \%$ fraction of all double electron captures are expected to originate from two K shell electrons [18]. The majority of these double K shell captures are expected to produce two characteristic X-rays per event.

We argue in this paper that the xenon-based high-pressure gas time projection chambers (TPCs) being developed by the NEXT collaboration for ${ }^{136} \mathrm{Xe} 0 \nu \beta \beta$ searches are ideally suited to also search for double electron capture in ${ }^{124} \mathrm{Xe}$, provided that a gas with sufficient ${ }^{124} \mathrm{Xe}$ fraction is used. From simulated signal data and background data taken with the NEXT-White detector we optimize the data selection cuts that provide the best sensitivity to the $2 \nu E C E C$ decay. These data are finally used to extrapolate the sensitivity to the NEXT-100 detector.

The paper is organized as follows. Section 2 describes the NEXT experimental program, with special emphasis in the NEXT-White detector used in this feasibility study. Section 3 describes the background data and signal simulation samples employed. The event selection and the sensitivity projections are presented in sections 4 and 5 , respectively. We conclude in section 6 .

\section{The NEXT experimental program}

The NEXT detectors rely on the technology of high-pressure (10-15 bar) xenon gas time projection chambers (TPCs) with electroluminescent (EL) amplification and optical readout. The gas TPC provides a homogeneous and low-density detector active volume. Electroluminescence provides a nearly noiseless amplification of the ionization electrons reaching the TPC anode, key for excellent energy resolution capabilities. The optical signals 

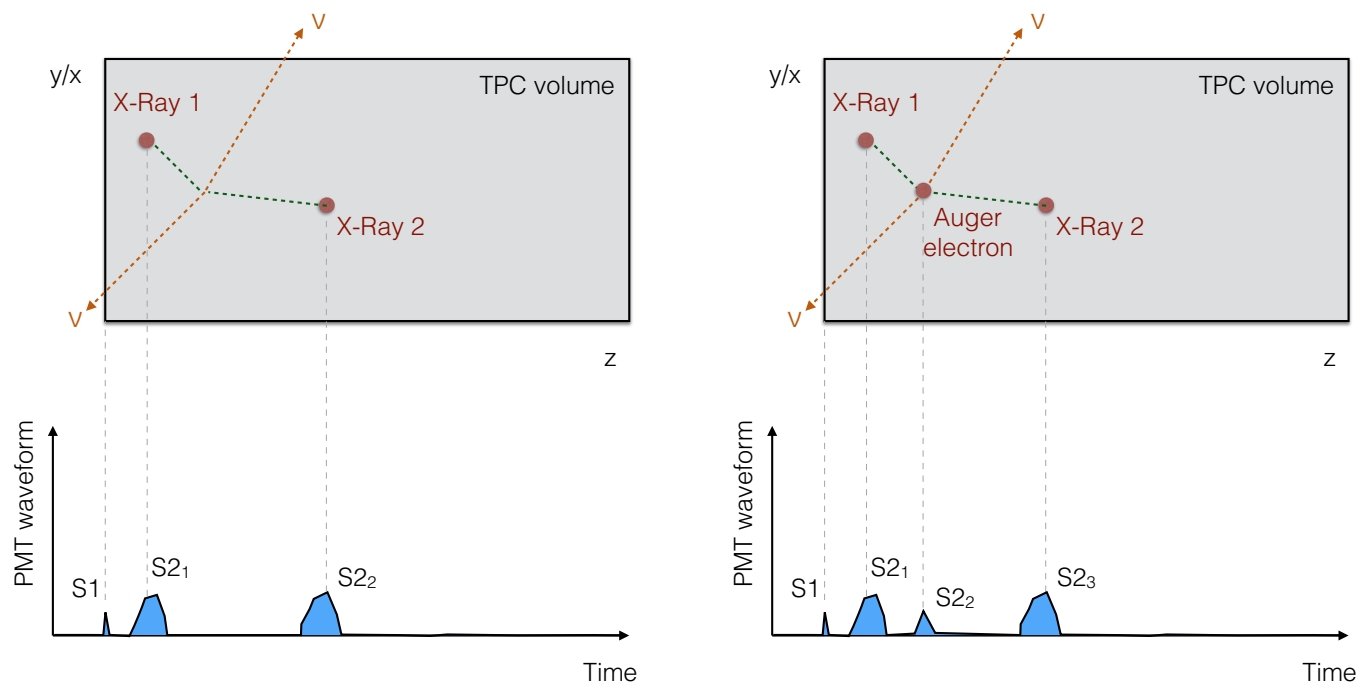

Figure 2. Schematic of $2 \nu E C E C$ signature in NEXT. Two golden samples are considered, with two (left panel) and three (right panel) separate energy depositions per event, respectively.

induced by xenon primary $\left(S_{1}\right)$ and electroluminescent $\left(S_{2}\right)$ scintillation are detected by readout planes at opposite ends of the detector's cylindrical volume, behind transparent cathode and anode planes, capable of providing a full 3D image of the events. The so-called tracking plane is composed of a large number of silicon photomultipliers (SiPMs) placed on a 2D lattice, and is located a few mm away from the anode and the EL region. The tracking plane detects the forward-going $S_{2}$ light, providing an unambiguous 2D image for each TPC time slice. The so-called energy plane is composed of photomultiplier tubes (PMTs) and is located behind the cathode, away from the EL region. The energy plane detects the nearly uniform backward-going $S_{2}$ light, providing an accurate energy measurement. The energy plane also detects the prompt $S_{1}$ light produced in the drift volume, enabling an accurate event $t_{0}$ determination.

The NEXT detection concept applied to $2 \nu E C E C$ signals is depicted schematically in figure 2. Each X-ray interaction in the active volume may give rise to a separate $S_{2}$ signal, some millimeters or centimeters away from the event vertex. For example, tellurium $K_{\alpha}$ $\mathrm{X}$-rays from $\mathrm{L} \rightarrow \mathrm{K}$ transitions produced by ${ }^{124} \mathrm{Xe}$ double electron capture, with an energy of $27.5 \mathrm{keV}$, have a linear attenuation coefficient of $1.6 \mathrm{~cm}$ in xenon at 10 bar. A weaker $S_{2}$ signal from Auger electrons at the event vertex may also be reconstructed. The $S_{2}$ signals have a pulse shape characteristic of point-like energy deposits, with a time width primarily affected by diffusion effects along ionization electron drift. Regardless of the number of $S_{2}$ signals, a single (narrower) $S_{1}$ signal characteristic of the full event energy deposition is also present in the event PMT waveforms.

The strengths of the NEXT approach are threefold. First, NEXT TPCs feature a better energy resolution in the energy region of interest compared to the liquid xenon scintillators or gas proportional counters listed in table 1. Second, NEXT provides full 3D position reconstruction capabilities to suppress external background events. This is also the case of liquid scintillators (which also provide xenon self-shielding), but only partially the 
case for the gas proportional counter of reference [9]. Third, the low density of the detector and its 3D imaging capabilities allow NEXT to spatially separate the X-ray conversions or Auger electron deposits for a significant fraction of all $2 \nu E C E C$ events. This is not possible in the higher-density liquid xenon, and only partially possible (that is, along the drift direction) in a gas proportional counter.

The first phase of the NEXT experimental program started in 2009 with the construction, commissioning and operation of two EL prototypes, NEXT-DEMO [19] and NEXT-DBDM [20], with xenon active masses of about $1 \mathrm{~kg}$. These prototypes demonstrated the robustness of the technology, its excellent energy resolution and its unique topological signature. The NEXT-White demonstrator [21], deploying $4 \mathrm{~kg}$ of xenon in its active volume, implements the second phase of the program. This detector has been operating underground at the Laboratorio Subterráneo de Canfranc (LSC, Spain) since 2016. NEXT-White is also the first radiopure detector in the NEXT series. Its main goals are a detailed assessment of the backgrounds for ${ }^{136} \mathrm{Xe}$ double beta decay searches, the measurement of the ${ }^{136} \mathrm{Xe} 2 \nu \beta \beta$ half-life and the characterization of the detector performance at energies close to the ${ }^{136} \mathrm{Xe}$ energy region of interest (about $2.5 \mathrm{MeV}$ ). The NEXT-100 detector, currently under construction, constitutes the third phase of the program. With a ${ }^{136} \mathrm{Xe}$ active mass approaching $100 \mathrm{~kg}$, NEXT-100 will perform the first sensitive $0 \nu \beta \beta$ search in xenon gas [22]. The fourth phase of the program contemplates tonne-scale xenon gas detectors. Two R\&D lines are being pursued in parallel. The NEXT technology can be scaled up to $0 \nu \beta \beta$ source masses in the tonne scale introducing several technological advancements already available [23, 24]. The NEXT collaboration is also pursuing a more radical approach to a tonne-scale experiment based on the efficient detection of the $\mathrm{Ba}^{++}$ion produced in the $0 \nu \beta \beta$ decay of ${ }^{136} \mathrm{Xe}$ using single-molecule fluorescence imaging (SMFI) [24-29].

\section{Data samples}

For a given ${ }^{124} \mathrm{Xe}$ mass in the detector, a reliable evaluation of the experimental sensitivity to ${ }^{124} \mathrm{Xe} 2 \nu E C E C$ relies on two factors: a good description of the backgrounds and a realistic estimate of the efficiency in reconstructing and selecting $2 \nu E C E C$ signal events. The lower the backgrond rate and the higher the signal efficiency, the better the ${ }^{124} \mathrm{Xe}$ $2 \nu E C E C$ sensitivity and the prospects for its observation. For this analysis, we combine a background dataset from the NEXT-White detector adding up to 5 months of data-taking (section 3.1) with a custom Monte-Carlo (MC) simulation of the ECEC signal (section 3.2).

\subsection{Background data sample}

The background sample used in this analysis was taken in 2019 between February 25th and July 10th and between September 13th and November 6th, as part of Run Va and Run Vb of the NEXT-White detector, which was filled with ${ }^{136}$ Xe-enriched gas. The amount of ${ }^{124} \mathrm{Xe}$ present in the source is negligible, as shown in figure 3, which makes this run configuration perfect for background characterization. The total accumulated exposure in this period is 125.9 days. 


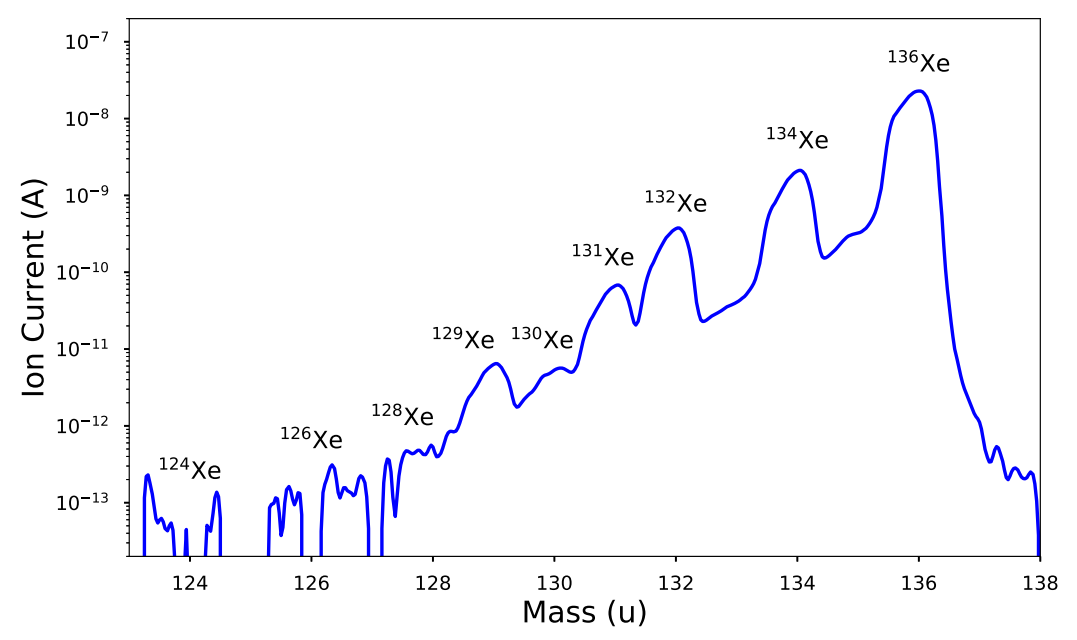

Figure 3. Isotopic composition of NEXT ${ }^{136}$ Xe-enriched gas.

The events considered in the analysis are those taken by the low-energy trigger of the detector, which is set to record events between approximately $8 \mathrm{keV}$ and $80 \mathrm{keV}$. This background dataset constitutes a total of $2.2 \times 10^{8}$ low-energy triggers. Detector conditions were stable during the whole data-taking period. The pressure was $10.13 \mathrm{bar}$, with voltages of $30 \mathrm{kV}$ and $7.7 \mathrm{kV}$ in the cathode and gate. The drift velocity of the electrons in the gas [30] remained fairly constant at $\sim 0.91 \mathrm{~mm} / \mu \mathrm{s}$. The electron lifetime during this period of datataking varied between 5 and $9.5 \mathrm{~ms}$, many times greater than the maximum drift time. The smooth variations in the detector conditions are accounted for using ${ }^{83 \mathrm{~m}} \mathrm{Kr}$ calibration data, which allows for a continuous monitoring of the electron lifetime and other detector properties [31].

Due to the low-energy nature of these events, the data are processed to obtain a full 3D point-like reconstruction for each separate energy deposit in the event. The waveforms from the PMTs are summed to identify $S_{1}$ and $S_{2}$ signals. The integral of each $S_{2}$ signal is used to determine the deposited energy in each site. The waveforms of the SiPM sensors are combined to perform a $2 \mathrm{D}$ reconstruction of each $S_{2}$ signal separately, which is in turn aggregated with the drift time to have one point-like $3 \mathrm{D}$ reconstruction per $S_{2}$ signal. The energy of each $S_{2}$ signal is finally corrected by lifetime and geometry effects and converted to $\mathrm{keV}$ on a run-by-run basis. For more details on NEXT-White low-energy data processing and calibration procedures, see reference [31].

\subsection{Signal simulation sample}

The only particles emitted in the ECEC process are neutrinos, which are undetectable. Hence, the simulation of the decay is focused on the daughter nucleus, which for ${ }^{124} \mathrm{Xe}$ is ${ }^{124} \mathrm{Te}$. Although electrons of any shell can be captured, $\mathrm{K}$ shell electrons have the highest probability. Thus, the aim is to study ${ }^{124} \mathrm{Te}$ atoms with a double $\mathrm{K}$ shell vacancy, which relax by emitting X-rays or Auger electrons. The simulation is based on the atomic relaxation package of Geant4 v10.04 [32]. Geant4 uses the Livermore Evaluation Atomic Data Library (EADL) [33], that contains data to describe the relaxation of atoms back 

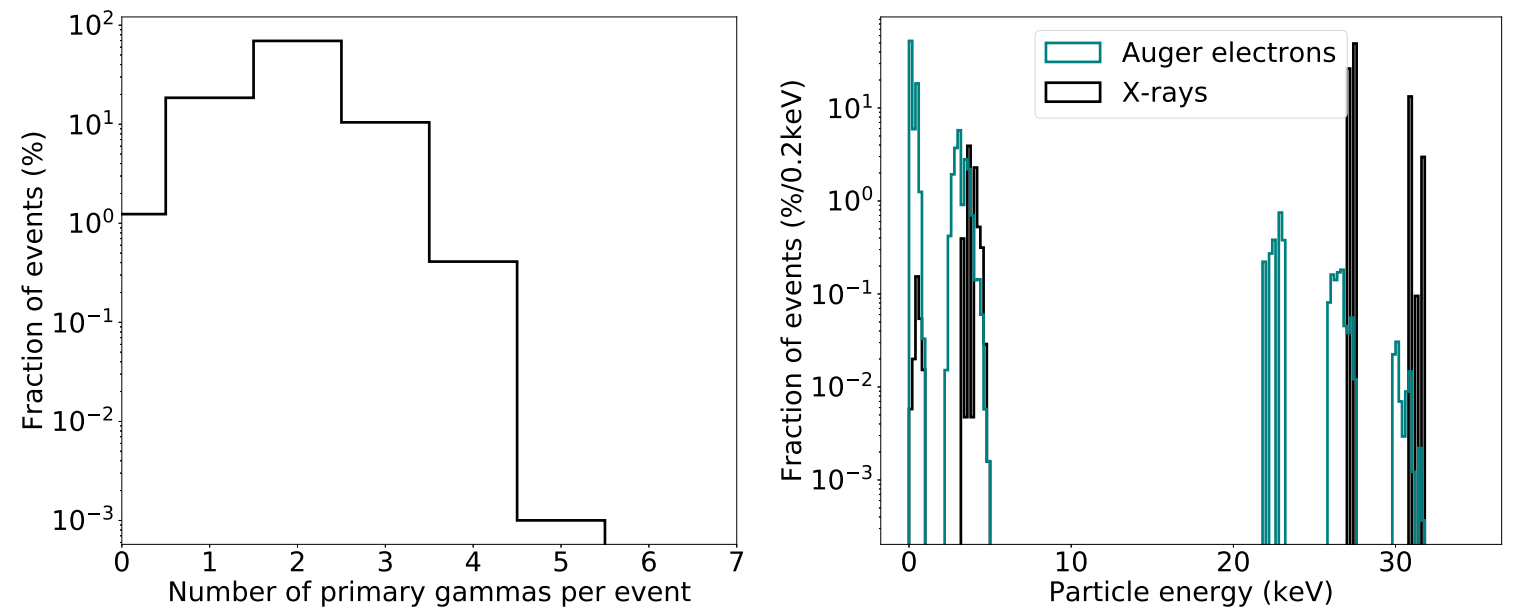

Figure 4. Monte-Carlo truth distributions of ${ }^{124} \mathrm{Xe} E C E C$ signal events. Left: number of primary gammas (X-rays) per simulated event. Right: distribution of simulated X-ray energies.

to neutrality after they are ionized. Since the package does not provide a mechanism to generate $E C E C$ events, we approximate our simulation by generating two independent ${ }^{124} \mathrm{Te}$ atoms with a single $\mathrm{K}$ shell vacancy and at the same spatial location per event, as done in reference [8].

Albeit the energy of two single $\mathrm{K}$ shell vacancies $(63.63 \mathrm{keV}$ [34]) is not the same of that of a double vacancy $(64.46 \mathrm{keV}$ [35]), we consider the difference, $0.8 \mathrm{keV}$, negligible.

Each atom in the simulation de-excites independently according to the X-ray and Auger emission probabilities. The observed emission probabilities, derived from the number of primary gammas in the simulation shown in the left panel of figure 4 are consistent with the fluorescence yield of ${ }^{124} \mathrm{Te}$ [36]. The energy of the emitted gammas matches also the atomic energy levels of the ${ }^{124} \mathrm{Te}$ atom, as shown on the right panel of figure 4 .

The interactions of electrons and gammas in the volume of the detector are simulated according to the G4EmStandardPhysics_option4 package of Geant4. Then, we simulate the electron drift (with realistic electron diffusion and negligible electron attachment), the light emission using parametrized models of the detector and the electronics response (including noise) to finally produce a set of sensor waveforms, equivalent to that of the real detector. These waveforms are then processed identically as detailed in section 3.1.

The full signal dataset is composed of $10^{5}$ events distributed homogeneously over the active volume of the NEXT-White detector.

\section{Event selection}

Signal and background events produce different signatures in the detector. At low energies $(<100 \mathrm{keV})$, the events in the chamber are primarily small-angle Compton scatterings of background gamma-rays, photoelectric interactions of low-energy gamma-rays, isolated xenon X-rays and, mostly, decays of metastable ${ }^{83 \mathrm{~m}} \mathrm{Kr}$ atoms introduced in the active volume as a calibration source [31]. These events are characterized by having a single- 
site interaction and thus producing one $S_{1}$ and one $S_{2}$ signals. On the other hand, the vast majority of $2 \nu E C E C$ events consist of at least two interactions, regardless of whether they come from X-rays or Auger electrons. This can be inferred for example from the left panel of figure 4, showing that approximately $99 \%$ of $2 \nu E C E C$ events are expected to produce at least one $\mathrm{X}$-ray. Hence, the event topology provides a key feature to discriminate background events from signal.

Notwithstanding, there are two cases in which this feature is not as useful. First, pile-up of different events in the DAQ window could in principle mimic the signature of a $E C E C$ event, if only one $S_{1}$ signal is reconstructed. However, the energies of the individual $S_{2}$ signals rarely match the ones expected from our signal events. Second, the two X-rays or Auger electrons from a $E C E C$ event can interact very close to the originating vertex, meaning that both signals merge into a single $S_{2}$, spoiling the topological signature of the process. A fraction of these events could be recovered by improving and customizing the reconstruction algorithms for this particular case, see discussion in section 5.2.

In order to optimize the signal and minimize the background, we perform a number of selections in the data. These selections are based both on topology and energy considerations. First, we reduce the data size by selecting a broad energy window around the region of interest (ROI), which eliminates most of the events coming from the ${ }^{83 \mathrm{~m}} \mathrm{Kr}$ source, see section 4.1. Second, we apply data quality cuts in section 4.2 . In section 4.3 we discuss the fiducial cuts. We then select the optimal energy ROI for the search, see section 4.4. We finally apply the multi-site event selection in section 4.5. Each selection is done separately and based on the optimization of the following figure of merit:

$$
\mathrm{FOM}=\frac{\varepsilon_{\mathrm{sig}}}{\sqrt{\varepsilon_{\mathrm{bkg}}}}
$$

where $\varepsilon$ is the relative efficiency of the cut defined as

$$
\varepsilon=\frac{\# \text { events after the cut }}{\# \text { events before the cut }} .
$$

A data reduction summary for signal and background events as a function of the various sequential cuts is given in table 2 and discussed in section 4.6.

\section{1 $\quad{ }^{83 \mathrm{~m}} \mathrm{Kr}$ veto requirement}

The ${ }^{83 \mathrm{~m}} \mathrm{Kr}$ calibration source used in the experiment produces $41.5 \mathrm{keV}$ single-site events that represent about $82 \%$ of the reconstructed low-energy events. The energy resolution of the NEXT-White detector at $41.5 \mathrm{keV}$ is approximately $1.8 \mathrm{keV}$ FWHM [31]. A $1 / \sqrt{E}$ extrapolation, demonstrated in [37], yields an expected energy resolution at $63.63 \mathrm{keV}$ of $2.2 \mathrm{keV}$. Thus, we can reduce the ${ }^{83 \mathrm{~m}} \mathrm{Kr}$ background to the minimum by requiring the energy of the entire event to be in a broad window above the ${ }^{83 \mathrm{~m}} \mathrm{Kr}$ peak.

As shown in figure 5, the energy spectrum is dominated by the ${ }^{83 \mathrm{~m}} \mathrm{Kr}$ peak at $41.5 \mathrm{keV}$ and, to a lesser extent, by the coincidence of two ${ }^{83 \mathrm{~m}} \mathrm{Kr}$ events in the same waveform at $83 \mathrm{keV}$. We remove these data from our sample by selecting the events with a total energy between $50 \mathrm{keV}<E_{\text {evt }}<80 \mathrm{keV}$. The combined requirement of successful event 


\begin{tabular}{|lcc|}
\hline Processing/selection step & $\begin{array}{c}\text { Fraction of } \\
\text { background data (\%) }\end{array}$ & $\begin{array}{c}\text { Fraction of } \\
\text { signal MC (\%) }\end{array}$ \\
\hline DAQ triggers or MC simulated events & 100 & 100 \\
Event reconstruction & $83.564 \pm 0.003$ & $89.10 \pm 0.10$ \\
$83 \mathrm{~m}$ Kr veto & $(40.53 \pm 0.04) \times 10^{-2}$ & $83.50 \pm 0.12$ \\
Data quality & $(13.99 \pm 0.02) \times 10^{-2}$ & $35.76 \pm 0.15$ \\
Fiducial & $(4.06 \pm 0.04) \times 10^{-3}$ & $25.42 \pm 0.14$ \\
Event energy & $(5.28 \pm 0.15) \times 10^{-4}$ & $24.39 \pm 0.13$ \\
Multi-site & $(1.15 \pm 0.07) \times 10^{-4}$ & $22.90 \pm 0.13$ \\
\hline
\end{tabular}

Table 2. Event selection summary. Cumulative signal and background efficiency as a function of the various event selection criteria.

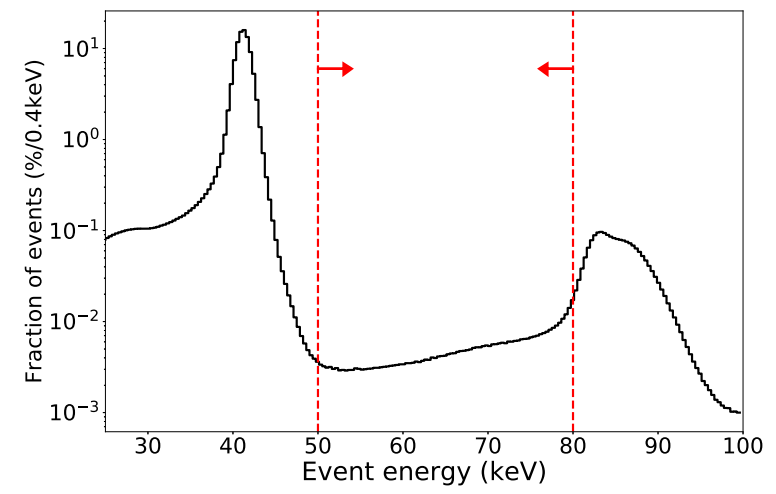

Figure 5. Event energy spectrum of fully reconstructed low-energy triggers for the full background dataset taken with the NEXT-White detector. Red lines indicate the broad energy window for the ${ }^{83 \mathrm{~m}} \mathrm{Kr}$ veto.

reconstruction plus ${ }^{83 \mathrm{~m}} \mathrm{Kr}$ veto keeps $0.4 \%$ of the background data, hence reducing the size of the data sample by a factor of 200 , while keeping $83.5 \%$ of the simulated $2 \nu E C E C$ signal events.

\subsection{Data quality selections}

Data quality criteria are imposed based on the multiplicity of reconstructed $S_{1}$ and $S_{2}$ signals per event. First, we remove all events with no or multiple $S_{1}$ signals. Events with no $S_{1}$ signals are not reconstructed along the drift coordinate. ${ }^{1}$ Therefore, they cannot be selected according to fiducial volume criteria nor their energy corrected for electron lifetime effects along drift. Events with more than one $S_{1}$ signal on the other hand introduce an ambiguity in the drift coordinate determination, resulting in the same limitations as events with no $S_{1}$ signals. Additionally, such events may originate from event pile-up. The single $S_{1}$ condition keeps $45.3 \%$ of the background data and $100 \%$ of the simulated signal.

\footnotetext{
${ }^{1}$ The drift time can also be estimated from the width of the $\mathrm{S} 2$ signal. This method is less accurate, but it might be required in larger detectors where the $S_{1}$ detection efficiency is smaller.
} 


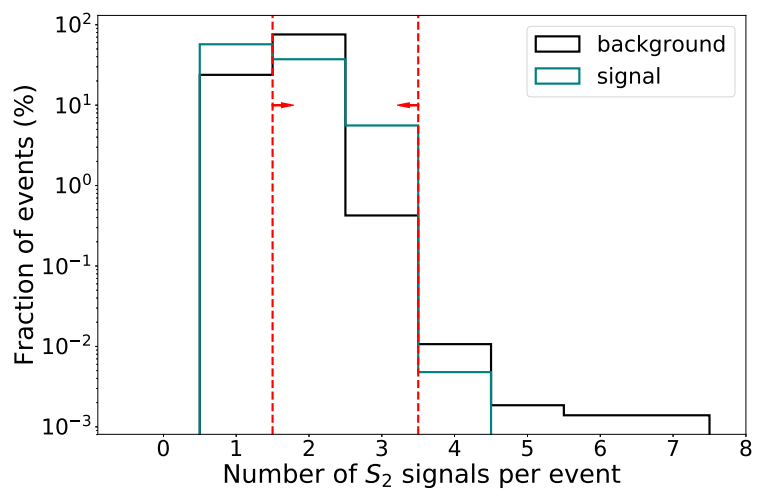

Figure 6. $S_{2}$ multiplicity per event for signal and background events after the ${ }^{83 \mathrm{~m}} \mathrm{Kr}$ veto. Red lines indicate the limits of the range of accepted values.

Second, we require each event to have either two or three $S_{2}$ signals. This condition is based on the topology of $2 \nu E C E C$ events, which should produce two or three separate energy depositions in most cases, see figures 2 and 4 . Thus, this requirement selects events in which at least two of them are spatially distinguishable along the drift coordinate. This cut keeps $42.8 \%$ of the signal events and $76 \%$ of the background events. Despite losing a significant fraction of signal events, for this study we assume that energy depositions that overlap in $\mathrm{Z}$ are not separable as doing so would require dedicated reconstruction algorithms as discussed in section 5.2. As shown in figure 6, the probability of reconstructing three $S_{2}$ signals per event after the ${ }^{83 \mathrm{~m}} \mathrm{Kr}$ veto is higher in signal than in background, while the opposite is true for two- $S_{2}$ events.

\subsection{Fiducial selection}

Signal events are homogeneously distributed over the entire active volume. On the other hand, background events tend to be reconstructed on the borders of the detector. Thus, we define a fiducial volume of the detector that maximizes the sensitivity to our search. An event is considered to pass the fiducial cut if and only if all of the $S_{2}$ signals satisfy the condition. Three cut variables are separately optimized to define the fiducial selection: the maximum radial position $R_{\max }$, the minimum drift distance $Z_{\min }$ and the maximum drift distance $Z_{\max }$ of all $S_{2}$ signals in the event. In order to obtain the optimal selection we evaluate the figure of merit in eq. (4.1) for each variable independently within a sensible range of values for the cut variables.

For the radial coordinate we only set an upper limit. The optimal value is found to be $R_{\max }=183.5 \mathrm{~mm}$, to be compared with the NEXT-White active volume radius of $R=198 \mathrm{~mm}$. The radial distributions of signal and background $S_{2}$ signals are shown in the left panel of figure 7, together with the cut position. With this cut we keep $76 \%$ of the simulated signal events and $9 \%$ of the background events.

For the longitudinal coordinate we set both a lower and an upper limit. The optimal values are $Z_{\min }=44.0 \mathrm{~mm}$ and $Z_{\max }=512.5 \mathrm{~mm}$, respectively. The TPC active volume boundaries along drift are $Z=0$ (anode) and $Z=530.3 \mathrm{~mm}$ (cathode). The drift distance 

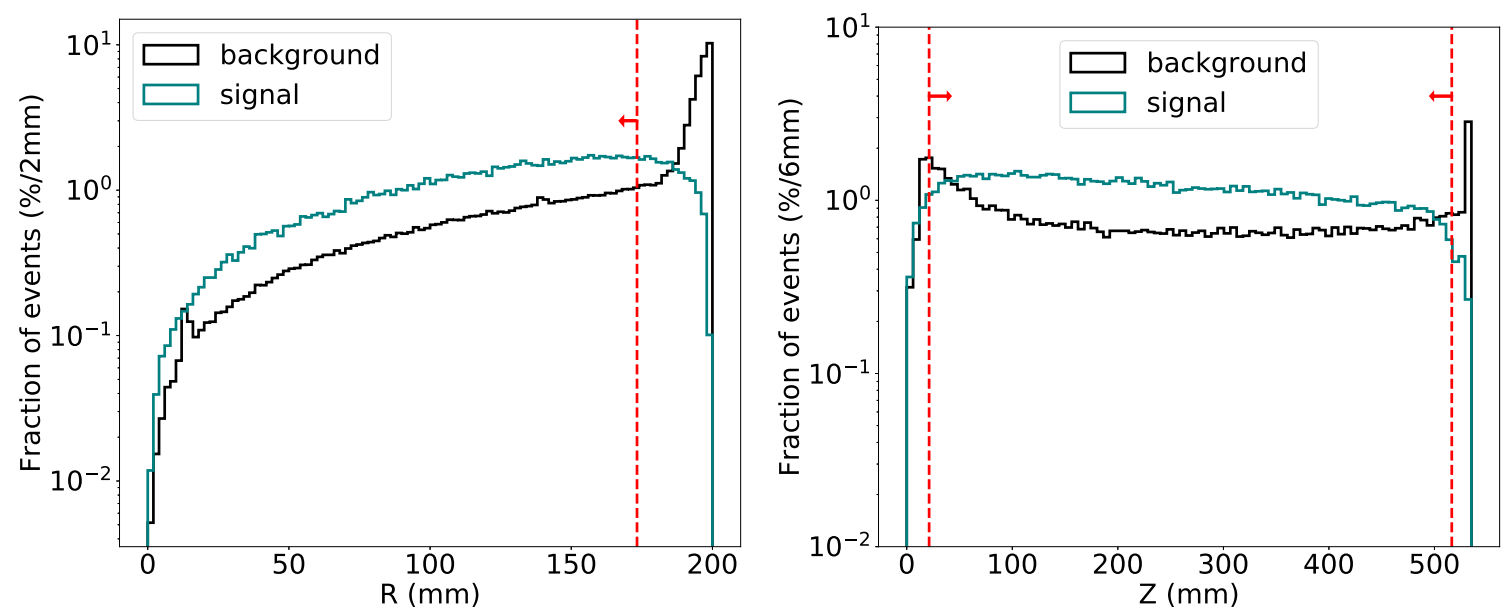

Figure 7. Event selection based on spatial information. One entry per reconstructed $S_{2}$ signal is shown. Left: signal and background distributions along the radial detector coordinate, with optimal $R_{\max }$ cut position (red line). Right: signal and background distributions along the drift coordinate and after the fiducial radius selection, with optimal $Z_{\min }$ and $Z_{\max }$ cut positions (red lines).

distributions of signal and background $S_{2}$ signals are shown in the right panel of figure 7 , together with the cut positions. Combining the lower and upper edges of the cut we end up keeping $93.5 \%$ of the signal events and $32 \%$ of the background events.

\subsection{Event energy selection}

As mentioned in section 3.2, the energy released in our simulated ${ }^{124} \mathrm{Xe} 2 \nu E C E C$ events is $63.63 \mathrm{keV}$. Considering the good energy resolution of the detector, it is rather convenient to restrict the energy ROI beyond that already done by the ${ }^{83 \mathrm{~m}} \mathrm{Kr}$ veto requirement of section 4.1.

The maximum and minimum allowed event energies are separately optimized to define the energy ROI $E_{\text {evt,min }}<E_{\text {evt }}<E_{\text {evt,max }}$. The optimal value for the upper limit is found to be $E_{\text {evt,max }}=64.6 \mathrm{keV}$, and for the lower limit $E_{\text {evt,min }}=56.4 \mathrm{keV}$. The combined requirement of both limits yields a $96 \%$ efficiency for signal events and a $13 \%$ efficiency for background events. Event energy distributions for signal and background fiducial events are shown in figure 8. As can be seen in the figure, the cut optimization selects a bimodal distribution for signal events: a primary peak corresponding to the full energy deposit being correctly reconstructed, plus a secondary peak where an energy of 4-5 keV either escapes the active volume or is not reconstructed. As shown in figure 4, X-ray lines of $4-5 \mathrm{keV}$ are indeed expected. Background events are found to have a flat energy distribution in the ROI.

\subsection{Multi-site selection}

We account for two signal sub-samples in our analysis: both a two- $S_{2}$ sub-sample and a three- $S_{2}$ sub-sample. For the two- $S_{2}$ sub-sample not all events are selected, but only those where both energy depositions in the event satisfy a certain energy requirement 


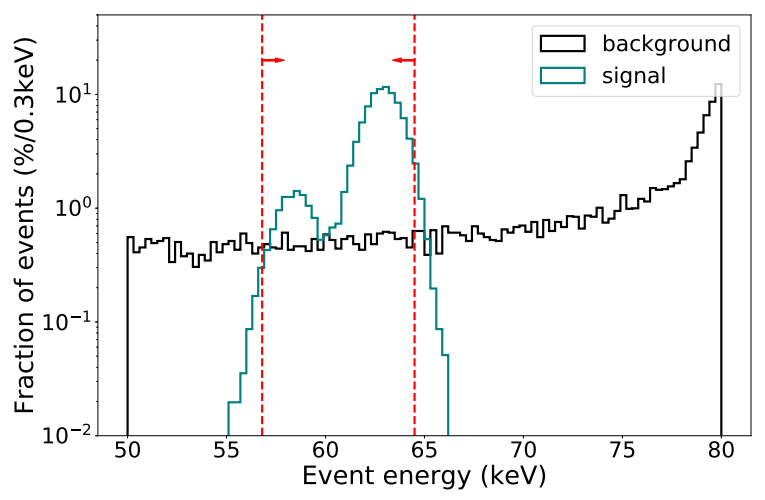

Figure 8. Event energy, $E_{\text {evt }}$, distribution for the signal and background datasets after fiducial selection, with optimal $E_{\text {evt }}$ cut positions (red lines).

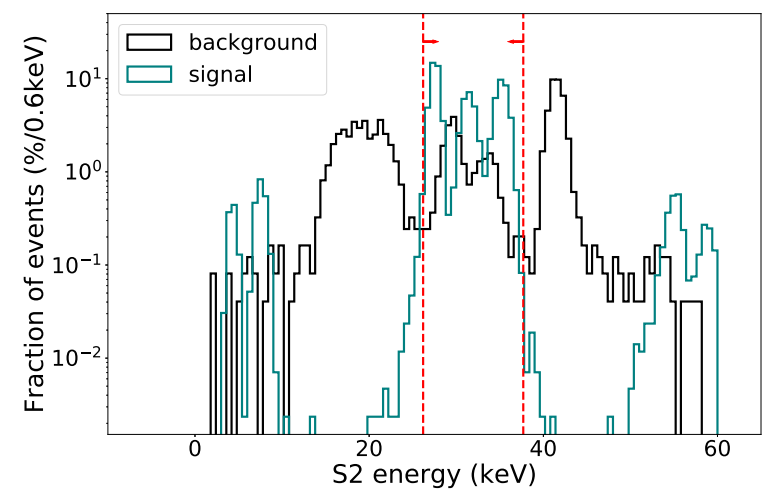

Figure 9. $S_{2}$ energy $E_{S_{2}}$ signal and background distribution in two- $S_{2}$ sub-sample, with optimal $E_{S_{2}}$ cut positions (red lines).

$E_{S_{2} \text {, min }}<E_{S_{2}}<E_{S_{2} \text {, max }}$. As observed in figure 4 (right), for signal we expect most isolated energy deposits to be due to tellurium $K_{\alpha}(\mathrm{L} \rightarrow \mathrm{K})$ X-rays, with a $S_{2}$ energy of $27.5 \mathrm{keV}$. As shown in the same figure, higher-energy X-rays of $31-32 \mathrm{keV}$ from initial electrons in outer shells are also possible.

Figure 9 shows the $S_{2}$ energy spectrum of the two- $S_{2}$ signal and background subsamples. After evaluating the figure of merit we obtain $E_{S_{2} \text {,min }}=26.0 \mathrm{keV}$ and $E_{S_{2} \text {,max }}=$ $37.3 \mathrm{keV}$ as the optimal limits. The combined effect of both limits keeps $94 \%$ of the signal events and $21.7 \%$ of the background events with $2 S_{2}$ signals.

As far as the three- $S_{2}$ sub-sample is concerned, no $E_{S_{2}}$ energy selection is performed and all events in the sub-sample are kept. As shown in figure 6, the probability to have three- $S_{2}$ events is an order of magnitude higher in signal than in background even without a $E_{S_{2}}$ requirement. After all cuts, the events with three- $S_{2}$ signals represent a $12.7 \%$ of the selected signal events and $0.5 \%$ of the selected background events.

\subsection{Event selection summary}

The cumulative efficiencies after each data processing step are shown in table 2 for both background data and signal MC events. We estimate a final efficiency of $(22.90 \pm 0.13) \%$ for 


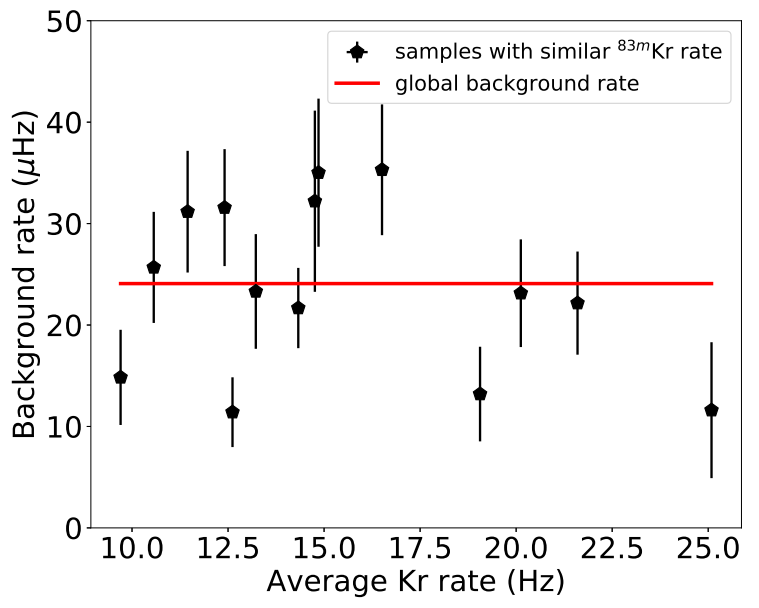

Figure 10. Background rate after all selections as a function of the ${ }^{83 \mathrm{~m}} \mathrm{Kr}$ rate for different samples of the full background dataset. The values oscillate around the overall background rate (solid line).

simulated double K shell captures, to be compared with an acceptance of $(1.15 \pm 0.07) \times 10^{-6}$ measured in ${ }^{124} \mathrm{Xe}$-depleted background data. The latter number corresponds to a total background rate of $24.7 \mu \mathrm{Hz}$, or 780 counts/yr.

We also analyze the dependence of the background rate after all selections on the initial rate of events. Since the background sample is dominated by the ${ }^{83 \mathrm{~m}} \mathrm{Kr}$ calibration source, we divide the full dataset into smaller samples with approximately the same ${ }^{83 \mathrm{~m}} \mathrm{Kr}$ rate. The optimized selections from the full dataset are then applied to these samples. Figure 10 shows the background rate after all selections as a function of the average ${ }^{83 \mathrm{~m}} \mathrm{Kr}$ rate in each sample. Clearly, the background rate does not depend on the ${ }^{83 \mathrm{~m}} \mathrm{Kr}$ rate, proving that the selection is robust and can be used in a variety of detector conditions.

While our data-driven approach to the background estimate implies that a full understanding of the background composition is a priori not known, much can be inferred from the background data distributions. On the one hand, figure 10 excludes ${ }^{83 \mathrm{~m}} \mathrm{Kr}$-related events as a dominant background source. On the other hand, figures 8 and 9 point to multisite interactions with a flat event energy spectrum in the region of interest, and where one of the energy deposits is a xenon K-shell X-ray at $29.7 \mathrm{keV}$ or $33.8 \mathrm{keV}$. Background events are therefore likely dominated by low-energy gamma rays produced by radioactive impurities in the detector materials, undergoing photoelectric absorption or by low-angle Compton scattering of higher energy gammas. We cannot determine which case is more likely to happen neither be certain about which gamma-ray emitting isotope is primarily responsible for the overall background rate. Based on findings from other experiments $[8,10]$ as well as from NEXT measurements at higher energies [38], ${ }^{60} \mathrm{Co},{ }^{40} \mathrm{~K}$, and isotopes in the ${ }^{238} \mathrm{U}$ and ${ }^{232} \mathrm{Th}$ decay chains are the likely main contributors.

\section{Sensitivity and precision projections}

The data-driven background evaluation and the corresponding $2 \nu E C E C$ signal efficiency study of section 4 have been done using NEXT-White data. However, our purpose is to 
study the feasibility of detecting $\mathrm{K}$ shell $2 \nu E C E C$ events in the forthcoming NEXT-100 detector (see section 2), if a significant ${ }^{124}$ Xe mass were added to its ${ }^{136}$ Xe-enriched xenon gas. The NEXT-100 detector is designed to hold approximately $100 \mathrm{~kg}$ of xenon with an enrichment fraction of $90 \%$ in the ${ }^{136} \mathrm{Xe}$ isotope. The natural abundance of ${ }^{124} \mathrm{Xe}$ is $0.095 \%$, or about $100 \mathrm{~g}$ of ${ }^{124} \mathrm{Xe}$ in $100 \mathrm{~kg}$ of natural xenon. In the ${ }^{136} \mathrm{Xe}$-enriched xenon to be used in NEXT-100, the amount of ${ }^{124} \mathrm{Xe}$ mass would be even less, as shown in figure 3. Such ${ }^{124} \mathrm{Xe}$ masses are too small for a competitive ${ }^{124} \mathrm{Xe} 2 \nu E C E C$ search. The objective of this study is therefore studying whether the addition of about $1 \mathrm{~kg}$ of highly ${ }^{124} \mathrm{Xe}$-enriched xenon to the $100 \mathrm{~kg}$ of ${ }^{136} \mathrm{Xe}$-enriched gas could yield a promising $2 \nu E C E C$ sensitivity in NEXT-100. In the following, we assume that NEXT-100 will contain $1 \mathrm{~kg}$ of ${ }^{124} \mathrm{Xe}$ in its active volume. The mixing of ${ }^{136} \mathrm{Xe}$ and ${ }^{124} \mathrm{Xe}$ would not affect the performance of the detector in any way, nor would it impact the program of ${ }^{136} \mathrm{Xe} \beta \beta$ searches in NEXT-100. The ${ }^{136} \mathrm{Xe}$ mass in the active volume would remain essentially the same after mixing this relatively small amount of ${ }^{124} \mathrm{Xe}$. The NEXT gas system is already prepared to perform such mixing operation, if a ${ }^{124} \mathrm{Xe}$ gas bottle were acquired.

The sensitivity to the ${ }^{124} \mathrm{Xe} 2 \nu E C E C$ half-life $T_{1 / 2}$, where both electrons are captured from the $\mathrm{K}$ shell, is computed as [4]:

$$
T_{1 / 2}=\ln 2 \cdot \frac{N_{A} \cdot \varepsilon_{s} \cdot M \cdot t}{W \cdot N_{s}}
$$

where $N_{A}$ the Avogadro's number, $\varepsilon_{s}=(22.90 \pm 0.13) \%$ is the $2 \nu E C E C$ signal efficiency estimated in section $4, M$ is the ${ }^{124} \mathrm{Xe}$ mass in the NEXT-100 active volume, $t$ is the exposure time, $W=123.9 \mathrm{~g} / \mathrm{mol}$ is the molar mass of the ${ }^{124} \mathrm{Xe}$ isotope, and $N_{s}$ is the maximum number of $2 \nu E C E C$ signal events that would be compatible with a backgroundonly measurement. Note that the average upper limit $N_{s}$ depends on the number of background events for an exposure time $t$, which has been estimated in section 4 , and on the confidence level value assumed. In the following, we use the customary $90 \%$ confidence level value. There are various prescriptions to evaluate $N_{s}$ given a certain background prediction. In this case, we use the standard Feldman-Cousins prescription [39]. The $2 \nu E C E C$ sensitivity of eq. (5.1) refers specifically to the double $\mathrm{K}$ shell capture process in ${ }^{124} \mathrm{Xe}$, as the event selection would be different for different capture configurations.

The XENON1T measurement yields a relatively high, $4.4 \sigma$, evidence for ${ }^{124} \mathrm{Xe}$ $2 \nu E C E C$. Therefore, in the following we also compute NEXT statistical precision in measuring the half-life assuming that XENON1T extracted central value is the true halflife of the process. Further assuming that the background prediction is perfectly known, and hence the only error in the signal extraction comes from the Poisson fluctuation in the total number of observed events, the relative statistical precision on the half-life for a given exposure can simply be written as:

$$
\delta T_{1 / 2} / T_{1 / 2}=\delta S / S=\sqrt{S+B} / S
$$

where $B$ and $S$ are the number of background and signal events passing all cuts, respectively. 


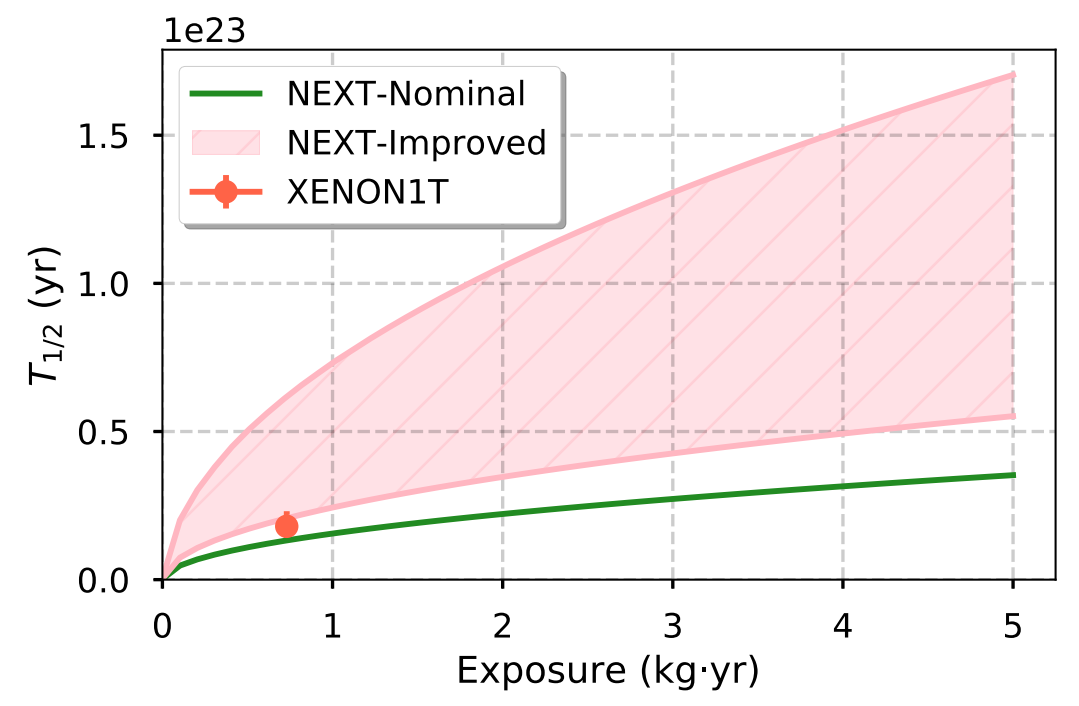

Figure 11. NEXT nominal (green curve) and improved (red band) half-life sensitivity to ${ }^{124} \mathrm{Xe}$ $2 \nu E C E C$ versus exposure, compared with the XENON1T measurement in reference [10] (red marker).

\subsection{Nominal sensitivity and precision}

The NEXT half-life sensitivity to ${ }^{124} \mathrm{Xe} 2 \nu E C E C$ is shown as a function of accumulated exposure (in $\mathrm{kg} \cdot \mathrm{yr}$ ) as the green line in figure 11 . A sensitivity of $1.6 \times 10^{22} \mathrm{yr}$ at $90 \% \mathrm{CL}$, comparable to the central value of the recent XENON1T measurement [10], is expected to be reached after an exposure of $1 \mathrm{~kg} \cdot \mathrm{yr}$ in NEXT-100. This is one year livetime of NEXT100 operated with $1 \mathrm{~kg}$ of ${ }^{124} \mathrm{Xe}$ mass in its active volume. In five years of operation in the same conditions, a sensitivity of $3.5 \times 10^{22} \mathrm{yr}$ at $90 \% \mathrm{CL}$ could be reached. We consider this nominal sensitivity to be conservative, as it assumes the background rate measured in NEXT-White and the $2 \nu E C E C$ signal efficiency achieved in this work, as presented in section 4.

NEXT relative statistical precision in the ${ }^{124} \mathrm{Xe} 2 \nu E C E C$ half-life measurement as a function of exposure, assuming a true value of $T_{1 / 2}=1.8 \times 10^{22} \mathrm{yr}$, is shown as the green curve in figure 12. For such nominal background rate and signal efficiency assumptions, a $30 \%$ precision is expected after a $5 \mathrm{~kg} \cdot \mathrm{yr}$ exposure.

In the following, we justify how more favorable assumptions for background rate and signal efficiency are plausible for NEXT-100, and we quantify their impact on the NEXT sensitivity and precision to double electron capture.

\subsection{Potential improvements}

The first potential improvement going from NEXT-White to NEXT-100 is the size of the detector. As the radius and length of the TPC are increased, the fiducial regions described in section 4.3 represent a larger fraction of the active volume. Thus, a larger fraction of the ${ }^{124} \mathrm{Xe}$ mass is kept, which translates into an increase in signal rate. 


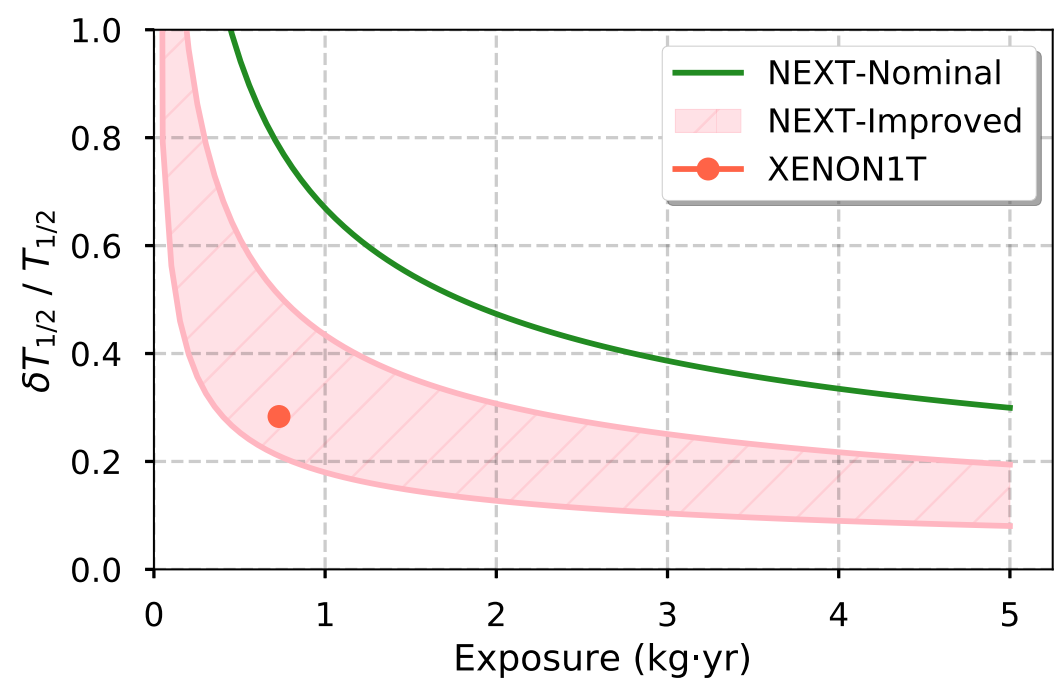

Figure 12. NEXT nominal (green curve) and improved (red band) relative precision in the measurement of the ${ }^{124} \mathrm{Xe} 2 \nu E C E C$ half-life as a function of exposure, assuming a true value of $T_{1 / 2}=1.8 \times 10^{22} \mathrm{yr}$ from [10]. The red marker shows the precision accomplished in [10].

The second improvement comes from changes in detector design that reduce the background rate. Compared to NEXT-White, NEXT-100 is being built from more radiopure materials and with a thicker inner shielding. In NEXT-100, the inner shielding is made of $12 \mathrm{~cm}$ of ultra-pure copper, to be compared with the $6 \mathrm{~cm}$ copper shielding of NEXT-White. The thicker shielding, together with the larger active volume, is expected to be particularly important to mitigate the low-energy $(\lesssim 100 \mathrm{keV})$ backgrounds relevant to $2 \nu E C E C$ searches.

Third, improvements in low-energy event reconstruction should significantly increase the $2 \nu E C E C$ signal efficiency in NEXT-100, and possibly also reduce the background rate. NEXT reconstruction algorithms used in section 3 and section 4 were developed for the higher-energy $\beta \beta$ searches. Dedicated low-energy reconstruction algorithms are likely to perform better. We show in figure 13 examples of PMT waveforms for simulated $2 \nu E C E C$ events that are incorrectly reconstructed by the current algorithms. As it is apparent from the waveforms, the reconstruction tends to merge into the same $S_{2}$ signal energy deposits that are visually separable from each other. This is due to the proximity of energy deposits along the drift direction and to the longitudinal diffusion, partially merging the nearby deposits together. As noted earlier (section 2), spatial separations of order 10$20 \mathrm{~mm}$ among energy deposits in the same event are common, in the $2 \nu E C E C$ case. In particular, a large inefficiency results from signal events that are mis-reconstructed as single $S_{2}$ signal events, which are discarded as background-like in our analysis. In addition to more sophisticated hit finding strategies, the performance of recently developed deconvolution algorithms indicates that the effect of electron diffusion can be reduced to a negligible level, which would also improve our ability to resolve close-by energy depositions. It is therefore plausible to obtain a large overall signal efficiency thanks to improvements in the reconstruction procedures combined with the larger active volume of NEXT-100. 

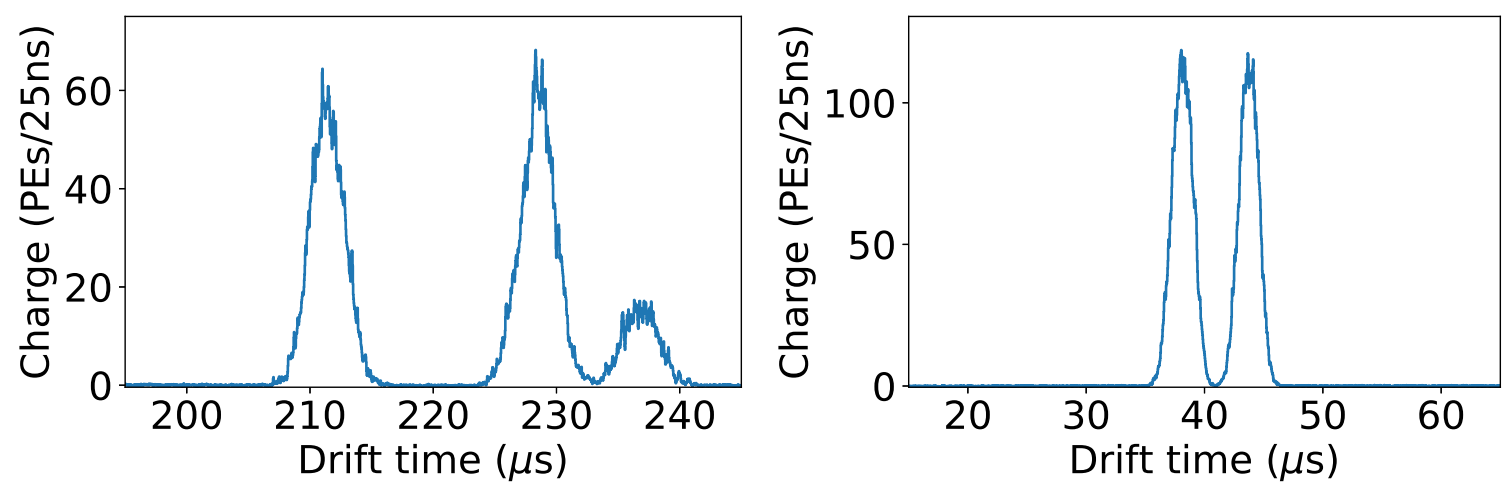

Figure 13. Examples of PMT waveforms of simulated $2 \nu E C E C$ signal events that are incorrectly identified by NEXT standard reconstruction algorithms. Left panel: event with three separate energy deposits, reconstructed as having two $S_{2}$ signals at times 211 and $228 \mu \mathrm{s}$, respectively. Right panel: event with two separate energy deposits, reconstructed as having one $S_{2}$ signal at time $38 \mu \mathrm{s}$.

Fourth, event selection might also be improved in NEXT-100. One possibility would be to adopt a multi-variate selection as opposed to sequential cuts as done in section 4 . In this case the selection would be optimized in a multi-dimensional space of observables, for example in $\left(R, Z, E_{\text {evt }}, E_{S_{2}}\right)$ space, accounting for correlations among them. On the other hand, another possibility to improve the signal/background event classification is by using deep learning techniques. This technique is already being used in NEXT for $0 \nu \beta \beta$ searches, yielding promising results [40].

We therefore believe that an improved half-life sensitivity in NEXT-100 is plausible, compared to the nominal sensitivity obtained from NEXT-White detector performance and backgrounds. In order to quantify this improvement, we provide an estimate of the signal efficiency assuming the optimal selection cuts described in section 4 applied on a simulated dataset of $2 \nu E C E C$ events in the NEXT-100 (as opposed to NEXT-White) detector geometry with negligible diffusion. In this baseline scenario, we obtain an improved signal efficiency of $35.82 \%$, to be compared with the nominal one of $22.90 \%$ in table 2 . The other major contributor to the sensitivity, the background rate, cannot be determined precisely. Nonetheless, a low-energy background rate reduction compared to NEXT-White is likely, as motivated above. Therefore for our optimistic scenario, we assume up to a factor-10 improvement on the background rate from the NEXT-White detector on top of the signal efficiency improvement.

This improved sensitivity is shown in figure 11 as a filled red band. The lower limit of the band is the baseline scenario with increased $2 \nu E C E C$ efficiency, $\varepsilon_{s}$, and minimal diffusion. The upper limit of the band assumes a factor of 10 reduction in background rate on top of that. Sensitivities in the $0.6-1.7 \times 10^{23} \mathrm{yr}$ range at $90 \% \mathrm{CL}$ and after 5 years of operation appear possible.

The improved assumptions on $2 \nu E C E C$ efficiency and background rate have also been used to quantify NEXT half-life measurement precision in a more optimistic scenario compared to section 5.1. As shown by the red band in figure 12, a significantly better precision in the $8-19 \%$ range would be achievable after a $5 \mathrm{~kg} \cdot \mathrm{yr}$ exposure. We also note that upcom- 
ing multi-tonne liquid xenon time projection chambers for direct dark matter detection, such as XENONnT [41], LZ [42] or PandaX-4T [43], should also be able to significantly improve the sensitivity and precision reach for ${ }^{124} \mathrm{Xe} 2 \nu E C E C$, compared to XENON1T. For a detailed discussion of the detection prospects for the second-order weak decays of ${ }^{124} \mathrm{Xe}$ in multi-tonne xenon time projection chambers, we refer to [44].

While this study focuses on ${ }^{124} \mathrm{Xe} 2 \nu E C E C$, an accurate measurement of the twoneutrino mode would set the stage for a sensitive search of the lepton number-violating $0 \nu E C E C$ mode of ${ }^{124} \mathrm{Xe}$, which would be the ultimate goal for double electron capture searches in NEXT.

\section{Conclusions}

The potential discovery of the Majorana nature of massive neutrinos via the observation of the neutrinoless decay modes of double beta decay processes is one of the most important questions in neutrino physics today. Neutrinoless double electron capture on proton-rich nuclei is a promising and alternative process compared to the far more exploited neutrinoless double $\beta^{-}$decay of neutron-rich nuclei. Yet, this process is significantly less understood from both the theoretical and the experimental points of view. The measurement of the Standard Model-allowed two-neutrino double electron capture is a first important step toward sensitive neutrinoless double electron capture searches. Two-neutrino double electron capture is a process in which two orbital electrons are simultaneously captured, typically from the $\mathrm{K}$ shell, by a proton-rich nucleus. The experimental signature is given by the emission of X-rays and Auger electrons from the de-excitation of the daughter atom.

In this paper, we establish that the high-pressure xenon gas TPC technology developed by the NEXT collaboration for ${ }^{136} \mathrm{Xe}$ double $\beta^{-}$decay searches is ideally suited to perform ${ }^{124} \mathrm{Xe}$ double electron capture searches as well. The reasons are the excellent energy resolution of the technology at the energy region of interest near $64 \mathrm{keV}$, its 3D imaging capabilities to suppress external backgrounds, and its capability to spatially separate the X-ray conversions or Auger electron deposits for a significant fraction of all double electron capture events. We have studied the feasibility to detect ${ }^{124} \mathrm{Xe}$ two-neutrino double electron capture in NEXT using actual NEXT-White background data and a detailed simulation of the signal in the same detector. The low-background data sample uses 125.9 days of NEXT-White low-energy triggers, originally designed for ${ }^{83 \mathrm{~m}} \mathrm{Kr}$ detector calibrations, with the detector filled with ${ }^{124} \mathrm{Xe}$-depleted xenon gas $\left(<10^{-5}\right.$ isotopic abundance). Both background data and simulated signal are reconstructed with NEXT standard reconstruction algorithms. An optimal event selection for ${ }^{124} \mathrm{Xe}$ two-neutrino double electron capture searches has been developed, maximizing the signal efficiency over the square root of background acceptance as the figure of merit. The event selection relies on the total event energy, on the multiplicity of xenon primary $\left(S_{1}\right)$ and charge-induced $\left(S_{2}\right)$ scintillation signals per event, and on the spatial location and energy of the individual energy depositions reconstructed in the event. As a result, a total background rate of $24.7 \mu \mathrm{Hz}$ ( 780 counts/yr) is measured in NEXT-White, for a total signal efficiency of $22.9 \%$. Extrapolating this background rate and signal efficiency to NEXT-100 and assuming it is operated with $1 \mathrm{~kg}$ of 
${ }^{124} \mathrm{Xe}$ in its active volume, an option that is technically feasible if a bottle with sufficient ${ }^{124}$ Xe-enriched gas quantity were procured, a sensitivity of $1.6 \times 10^{22} \mathrm{yr}$ at $90 \%$ CL could be obtained after one year of operations. This sensitivity is comparable to the recent measurement of ${ }^{124} \mathrm{Xe}$ by the XENON1T collaboration, $(1.8 \pm 0.5) \times 10^{22} \mathrm{yr}[10]$. We use this result as a basis to assess the case for a ${ }^{124} \mathrm{Xe}$ two-neutrino double electron capture measurement in the NEXT-100 detector, currently under construction. We expect that a dedicated double electron capture reconstruction capable of removing diffusion effects and better discerning separate energy deposits could increase the total signal efficiency to $35.82 \%$. With this improvement alone, we predict a two-neutrino double electron capture sensitivity of $6 \times 10^{22}$ yr after 5 years of operation, for the same background rate (780 counts/yr) and the same ${ }^{124}$ Xe active mass $(1 \mathrm{~kg})$. Assuming that XENON1T extracted central value is the true half-life of the process, a relative statistical precision of $19 \%$ in the half-life measurement is expected in NEXT-100 after 5 years. Other NEXT-100 detector design and analysis improvements compared to NEXT-White may yield further improvements in background reduction, resulting in even better sensitivity and precision.

\section{Acknowledgments}

The NEXT collaboration acknowledges support from the following agencies and institutions: the European Research Council (ERC) under the Advanced Grant 339787-NEXT; the European Union's Framework Programme for Research and Innovation Horizon 2020 (2014-2020) under the Marie Skłodowska-Curie Grant Agreements No. 674896, 690575 and 740055; the Ministerio de Economía y Competitividad and the Ministerio de Ciencia, Innovación y Universidades of Spain under grants FIS2014-53371-C04, RTI2018-095979, the Severo Ochoa Program grants SEV-2014-0398 and CEX2018-000867-S, and the María de Maeztu Program MDM-2016-0692; the GVA of Spain under grants PROMETEO/2016/120 and SEJI/2017/011; the Portuguese FCT under project PTDC/FIS-NUC/2525/2014, under project UID/FIS/04559/2013 to fund the activities of LIBPhys, and under grants PD/BD/105921/2014, SFRH/BPD/109180/2015 and SFRH/BPD/76842/2011; the U.S. Department of Energy under contracts number DE-AC02-06CH11357 (Argonne National Laboratory), DE-AC02-07CH11359 (Fermi National Accelerator Laboratory), DE-FG0213ER42020 (Texas A\&M) and DE-SC0019223/DE-SC0019054 (University of Texas at Arlington); and the University of Texas at Arlington. DGD acknowledges Ramon y Cajal program (Spain) under contract number RYC-2015-18820. We also warmly acknowledge the Laboratori Nazionali del Gran Sasso (LNGS) and the Dark Side collaboration for their help with TPB coating of various parts of the NEXT-White TPC. Finally, we are grateful to the Laboratorio Subterráneo de Canfranc for hosting and supporting the NEXT experiment.

Open Access. This article is distributed under the terms of the Creative Commons Attribution License (CC-BY 4.0), which permits any use, distribution and reproduction in any medium, provided the original author(s) and source are credited. 


\section{References}

[1] Super-Kamiokande collaboration, Evidence for oscillation of atmospheric neutrinos, Phys. Rev. Lett. 81 (1998) 1562 [hep-ex/9807003] [INSPIRE].

[2] SNO collaboration, Measurement of the rate of $\nu_{e}+d \rightarrow p+p+e^{-}$interactions produced by ${ }^{8}$ B solar neutrinos at the Sudbury Neutrino Observatory, Phys. Rev. Lett. 87 (2001) 071301 [nucl-ex/0106015] [INSPIRE].

[3] SNO collaboration, Direct evidence for neutrino flavor transformation from neutral current interactions in the Sudbury Neutrino Observatory, Phys. Rev. Lett. 89 (2002) 011301 [nucl-ex/0204008] [INSPIRE].

[4] J.J. Gomez-Cadenas, J. Martin-Albo, M. Mezzetto, F. Monrabal and M. Sorel, The Search for neutrinoless double beta decay, Riv. Nuovo Cim. 35 (2012) 29 [arXiv:1109.5515] [INSPIRE].

[5] R.G. Winter, Double K Capture and Single K Capture with Positron Emission, Phys. Rev. 100 (1955) 142 [INSPIRE].

[6] J. Bernabeu, A. De Rujula and C. Jarlskog, Neutrinoless Double Electron Capture as a Tool to Measure the $\nu_{e}$ Mass, Nucl. Phys. B 223 (1983) 15 [InSPIRE].

[7] XENON collaboration, Search for two-neutrino double electron capture of ${ }^{124}$ Xe with XENON100, Phys. Rev. C 95 (2017) 024605 [arXiv: 1609.03354] [InSPIRE].

[8] XMASS collaboration, Improved search for two-neutrino double electron capture on ${ }^{124} X e$ and ${ }^{126}$ Xe using particle identification in XMASS-I, Prog. Theor. Exp. Phys. 2018 (2018) $053 \mathrm{D} 03$ [arXiv: 1801.03251] [INSPIRE].

[9] Y.M. Gavriljuk et al., $2 K$-Capture in ${ }^{124}$ Xe: Results of Data Processing for an Exposure of $37.7 \mathrm{~kg}$ day, Phys. Part. Nucl. 49 (2018) 563 [arXiv: 1806.03060] [INSPIRE].

[10] XENON collaboration, Observation of two-neutrino double electron capture in ${ }^{124}$ Xe with XENON1T, Nature 568 (2019) 532 [arXiv:1904.11002] [INSPIRE].

[11] A.P. Meshik, C.M. Hohenberg, O.V. Pravdivtseva and Y.S. Kapusta, Weak decay of ${ }^{130} B a$ and ${ }^{132}$ Ba: Geochemical measurements, Phys. Rev. C 64 (2001) 035205 [InSPIRE].

[12] M. Pujol, B. Marty, P. Burnard and P. Philippot, Xenon in archean barite: Weak decay of ${ }^{130}$ Ba, mass-dependent isotopic fractionation and implication for barite formation, Geochim. Cosmochim. Acta 73 (2009) 6834.

[13] S.S. Ratkevich et al., Comparative study of the double K-shell-vacancy production in singleand double-electron capture decay, Phys. Rev. C 96 (2017) 065502 [arXiv:1707.07171] [INSPIRE].

[14] J.R. de Laeter et al., Atomic weights of the elements. Review 2000, Pure Appl. Chem. 75 (2003) 683.

[15] J. Suhonen, Double beta decays of ${ }^{124}$ Xe investigated in the QRPA framework, J. Phys. G 40 (2013) 075102 [INSPIRE].

[16] P. Pirinen and J. Suhonen, Systematic approach to $\beta$ and $2 \nu \beta \beta$ decays of mass $A=100-136$ nuclei, Phys. Rev. C 91 (2015) 054309 [inSPIRE].

[17] E.A. Coello Pérez, J. Menéndez and A. Schwenk, Two-neutrino double electron capture on ${ }^{124}$ Xe based on an effective theory and the nuclear shell model, Phys. Lett. B 797 (2019) 134885 [arXiv: 1809.04443] [INSPIRE]. 
[18] M. Doi and T. Kotani, Neutrinoless modes of double beta decay, Prog. Theor. Phys. 89 (1993) 139 [INSPIRE].

[19] NEXT collaboration, Operation and first results of the NEXT-DEMO prototype using a silicon photomultiplier tracking array, 2013 JINST 8 P09011 [arXiv:1306. 0471] [INSPIRE].

[20] NEXT collaboration, Near-Intrinsic Energy Resolution for 30 to $662 \mathrm{keV}$ Gamma Rays in a High Pressure Xenon Electroluminescent TPC, Nucl. Instrum. Meth. A 708 (2013) 101 [arXiv: 1211.4474] [INSPIRE].

[21] NEXT collaboration, The Next White (NEW) Detector, 2018 JINST 13 P12010 [arXiv: 1804.02409] [INSPIRE].

[22] NEXT collaboration, Sensitivity of NEXT-100 to Neutrinoless Double Beta Decay, JHEP 05 (2016) 159 [arXiv: 1511.09246] [INSPIRE].

[23] NEXT collaboration, Sensitivity of a tonne-scale NEXT detector for neutrinoless double beta decay searches, arXiv:2005.06467 [INSPIRE].

[24] APPEC Committee collaboration, Double Beta Decay APPEC Committee Report, arXiv: 1910.04688 [INSPIRE].

[25] D.R. Nygren, Detecting the barium daughter in ${ }^{136}$ Xe $0-\nu \beta \beta$ decay using single-molecule fluorescence imaging techniques, J. Phys. Conf. Ser. 650 (2015) 012002 [inSPIRE].

[26] B.J.P. Jones, A.D. McDonald and D.R. Nygren, Single Molecule Fluorescence Imaging as a Technique for Barium Tagging in Neutrinoless Double Beta Decay, 2016 JINST 11 P12011 [arXiv: 1609.04019] [INSPIRE].

[27] NEXT collaboration, Demonstration of Single Barium Ion Sensitivity for Neutrinoless Double Beta Decay using Single Molecule Fluorescence Imaging, Phys. Rev. Lett. 120 (2018) 132504 [arXiv: 1711.04782] [INSPIRE].

[28] P. Thapa et al., Barium Chemosensors with Dry-Phase Fluorescence for Neutrinoless Double Beta Decay, Sci. Rep. 9 (2019) 15097 [arXiv:1904.05901] [InSPIRE].

[29] I. Rivilla et al., Towards a background-free neutrinoless double beta decay experiment based on a fluorescent bicolor sensor, arXiv:1909.02782 [INSPIRE].

[30] NEXT collaboration, Electron drift properties in high pressure gaseous xenon, 2018 JINST 13 P07013 [arXiv: 1804.01680] [INSPIRE].

[31] NEXT collaboration, Calibration of the NEXT-White detector using ${ }^{83 m} \mathrm{Kr}$ decays, 2018 JINST 13 P10014 [arXiv: 1804.01780] [INSPIRE].

[32] GEANT4 collaboration, GEANT4 - a simulation toolkit, Nucl. Instrum. Meth. A $\mathbf{5 0 6}$ (2003) 250 [INSPIRE].

[33] S.T. Perkins, M.H. Chen, D.E. Cullen and J.H. Hubbell, Tables and graphs of atomic subshell and relaxation data derived from the LLNL Evaluated Atomic Data Library (EADL), $Z=1-100$, Lawrence Livermore National Laboratory, Livermore CA U.S.A. (1991) [UCRL-50400-V-30].

[34] J.A. Bearden and A.F. Burr, Reevaluation of X-Ray Atomic Energy Levels, Rev. Mod. Phys. 39 (1967) 125 [INSPIRE].

[35] D.A. Nesterenko et al., Double- $\beta$ transformations in isobaric triplets with mass numbers $A=124,130$, and 136, Phys. Rev. C 86 (2012) 044313 [inSPIRE]. 
[36] R. Firestone, V. Shirley, C. Baglin, S. Chu and J. Zipkin, Table of Isotopes, eight edition, John Wiley and Sons, Inc. (1997).

[37] NEXT collaboration, Energy calibration of the NEXT-White detector with $1 \%$ resolution near $Q_{\beta \beta}$ of ${ }^{136} \mathrm{Xe}, \mathrm{JHEP} 10$ (2019) 230 [arXiv:1905.13110] [INSPIRE].

[38] NEXT collaboration, Radiogenic Backgrounds in the NEXT Double Beta Decay Experiment, JHEP 10 (2019) 051 [arXiv:1905.13625] [INSPIRE].

[39] G.J. Feldman and R.D. Cousins, A Unified approach to the classical statistical analysis of small signals, Phys. Rev. D 57 (1998) 3873 [physics/9711021] [INSPIRE].

[40] NEXT collaboration, Background rejection in NEXT using deep neural networks, 2017 JINST 12 T01004 [arXiv:1609.06202] [INSPIRE].

[41] E. Aprile et al., Projected WIMP sensitivity of the XENONnT dark matter experiment, JCAP 11 (2020) 031 [arXiv: 2007.08796] [INSPIRE].

[42] LZ collaboration, The LUX-ZEPLIN (LZ) Experiment, Nucl. Instrum. Meth. A 953 (2020) 163047 [arXiv: 1910.09124] [INSPIRE].

[43] PandaX collaboration, Dark matter direct search sensitivity of the PandaX-4T experiment, Sci. China Phys. Mech. Astron. 62 (2019) 31011 [arXiv:1806.02229] [InSPIRE].

[44] C. Wittweg, B. Lenardo, A. Fieguth and C. Weinheimer, Detection prospects for the second-order weak decays of ${ }^{124} \mathrm{Xe}$ in multi-tonne xenon time projection chambers, Eur. Phys. J. C 80 (2020) 1161 [arXiv:2002.04239] [INSPIRE]. 